\title{
Ultrafast Dynamics of Photoionized Acetylene
}

\author{
Mohamed El-Amine Madjet, ${ }^{1}$ Oriol Vendrell, ${ }^{1, *}$ and Robin Santra ${ }^{1,2}$ \\ ${ }^{1}$ Center for Free-Electron Laser Science, DESY, Notkestrasse 85, D-22607 Hamburg, Germany \\ ${ }^{2}$ Department of Physics, University of Hamburg, D-20355 Hamburg, Germany
}

(Received 29 July 2011; published 20 December 2011)

\begin{abstract}
Acetylene cations $[\mathrm{HCCH}]^{+}$produced in the $A^{2} \Sigma_{g}^{+}$state by extreme ultraviolet (XUV) photoionization are investigated theoretically, based on a mixed quantum-classical approach. We show that the decay of the $A^{2} \Sigma_{g}^{+}$state occurs via both ultrafast isomerization and nonradiative electronic relaxation. We find a time scale for hydrogen migration and electronic decay of about $60 \mathrm{fs}$, in good agreement with recent XUV-pump/XUV-probe time-resolved experiments on the same system [Phys. Rev. Lett. 105, 263002 (2010)]. Moreover, we predict an efficient vibrational energy redistribution mechanism that quickly transfers excess energy from the isomerization coordinates to slower modes in a few hundred femtoseconds, leading to a partial regeneration of acetylenelike conformations.
\end{abstract}

PACS numbers: 33.80.Eh, 82.20.Gk

Photoisomerization is a fundamental process in chemistry and biology in which conformational changes between isomers are induced by light absorption. One of the most fascinating biological processes is the photoisomerization of the retinal molecule [1]. Absorption of light causes this molecule to change its conformation from 11-cis to alltrans, representing the primary photochemical event in vision. Photoisomerization reactions are complex chemical events, in which electronic excitation is channeled into specific vibrational modes that lead the system to different regions of conformational space before the excess energy is irreversibly dissipated as heat [2]. Dramatic examples include the conversion of light energy into motion in driving micromachines or nanomachines [3]. However, already molecules with a few atoms exhibit complex reactivity and irreversible energy redistribution processes occurring in a few hundreds of femtoseconds after localized excitations [4].

Because of its fundamental nature, the isomerization of acetylene $[\mathrm{HCCH}]^{+}$into vinylidene $[\mathrm{HHCC}]^{+}$has attracted extensive experimental and theoretical interest [5-12]. Acetylene is one of the smallest organic molecules. However, it has a rich electronic structure characterized by both $\sigma$ and $\pi$ bonding, and it has six internal vibrational degrees of freedom with vibrational frequencies in the ground electronic state spanning the range from 500 to $3200 \mathrm{~cm}^{-1}$. Especially interesting in acetylene is the ultrafast dynamics of the monocation produced in the $A^{2} \Sigma_{g}^{+}$ electronic state, for which the decay mechanism has been a matter of controversy over many years. It is already well known that this state has an extremely short lifetime [13]. Several decay mechanisms have been proposed in the literature [9], in particular, the decay via hydrogen migration $[5,8]$ or conical intersections $[11,14]$. Based on theoretical predictions, Chambaud et al. suggested an isomerization process from acetylene to vinylidene to be the main decay mechanism [8]. In the framework of a vibronic-coupling model, Gillen et al. investigated the dynamics and spectroscopy of the $X^{2} \Pi_{u}$ and $A^{2} \Sigma_{g}^{+}$states of acetylene cation [11]. They explained the short lifetime of the $A^{2} \Sigma_{g}^{+}$state in terms of an ultrafast internalconversion process caused by the conical intersection of $A^{2} \Sigma_{g}^{+}$with the $X^{2} \Pi_{u}$ state. With recent progress in the generation of ultrashort extreme ultraviolet (XUV) pulses by high-harmonic generation [15] or by free-electron lasers [16], it has become possible to follow the dynamics of ionized systems on femtosecond time scales. Recently, Jiang et al. reported a time-resolved experiment on the ultrafast isomerization of the acetylene cation in a set of ground-breaking XUV-pump/XUV-probe measurements conducted at FLASH, the free-electron laser in Hamburg [7]. The isomerization time was estimated to be $52 \pm 15 \mathrm{fs}$ based on the $\mathrm{CH}_{2}^{+}$production yield as a function of the pump-probe delay, providing direct evidence of the isomerization channel as the main decay mechanism of the $A^{2} \Sigma_{g}^{+}$state.

In this Letter, using a mixed quantum-classical approach, we present a complete time-resolved picture of the dynamics after population of the $A^{2} \Sigma_{g}^{+}$state by absorption of an XUV photon and emission of a valence electron. We find that ultrafast hydrogen migration goes hand in hand with an ultrafast nonradiative decay of the $A^{2} \Sigma_{g}^{+}$state to the $X^{2} \Pi_{u}$ state via conical intersections, and both processes take place in the sub-100-fs regime. This finding unifies the two most prominent explanations for the fast decay of the $A^{2} \Sigma_{g}^{+}$within the same theoretical framework. The molecular cations that have electronically decayed contain a large amount of vibrational energy. We find that the skeletal carbon-carbon stretching mode can efficiently take a large fraction of that energy in 300$400 \mathrm{fs}$, acting as a reservoir and cooling down the isomerization coordinates. This internal vibrational energy redistribution (IVR) process is responsible for a partial return to acetylenelike conformations in less than half a picosecond 
after ionization. The reported simulations are based on a mixed quantum-classical approach, in which the nuclear motions are described classically by Newton's equations while the electrons are treated quantum mechanically. The potential energy surfaces (PESs) of the cationic states are computed on the fly at the one-hole level as $E_{\mathrm{HF}}-\varepsilon_{i}$, where $E_{\mathrm{HF}}$ is the Hartree-Fock (HF) energy of the neutral acetylene and $\epsilon_{i}$ is the energy of occupied orbital $\phi_{i}$. This Koopmans' theorem based treatment of the cationic PESs has the advantage of providing, at a low cost, qualitatively good potentials for outer valence cationic states such as those of interest here. This makes it possible to propagate trajectories for many vibrational periods and gives access to vibrational energy redistribution processes occurring between fast and slow degrees of freedom. An important advantage of the approach in extending it to larger systems is given by the fact that it relies solely on quantities available from ground-state HF calculations. In the present case, a full quantum treatment of the nuclear dynamics would be very challenging due to large amplitude motions and large vibrational energies involved. Newton's equations were integrated with the velocity-Verlet algorithm and a time step of 0.25 fs. The initial conditions for each trajectory were sampled from a Boltzmann distribution at $T=10 \mathrm{~K}$ [17] of positions and velocities on the ground electronic PES of the neutral system. The sogenerated initial conditions were used to start trajectories on the cationic surfaces at $t=0$. One hundred trajectories were used in the calculations of ensemble-averaged quantities. In order to describe nonradiative electronic-state transitions resulting from the breakdown of the Born-Oppenheimer approximation, a trajectory surfacehopping (TSH) algorithm was used [18]. The transition probabilities for the TSH were computed based on the Landau-Zener semiclassical approximation [19,20]. The implementation used here closely follows that of Jones et al. [20].

Neutral acetylene in its ground state in a linear conformation is described by the electronic configuration $1 \sigma_{g}^{2}$ $1 \sigma_{u}^{2} 2 \sigma_{g}^{2} 2 \sigma_{u}^{2} 3 \sigma_{g}^{2} 1 \pi_{u}^{4}$. With $38 \mathrm{eV}$ photons, as used in the experiment by Jiang et al. [7], electrons from one of the following occupied orbitals can be removed: $1 \pi_{u}, 3 \sigma_{g}$, $2 \sigma_{u}, 2 \sigma_{g}$, which lead to states $X^{2} \Pi_{u}, A^{2} \Sigma_{g}^{+}, B^{2} \Sigma_{u}^{+}, C^{2} \Sigma_{g}^{+}$, respectively. Throughout this Letter we refer to the electronic states by their nomenclature in the linear structure irrespective of molecular geometry. The probability of populating the $A^{2} \Sigma_{g}^{+}$state with $40 \mathrm{eV}$ photons is about $28 \%$ [21]. Although simulations were run starting on each of the five possible PESs accessed by removal of a valence electron, as expected only in the $A^{2} \Sigma_{g}^{+}$state ultrafast conformational changes are observed. This is in good agreement with the reported relative yields of $\mathrm{C}^{+}+\mathrm{CH}_{2}^{+}$ and $\mathrm{CH}^{+}+\mathrm{CH}^{+}$(see Fig. 2 in Ref. [7]), the former channel being clearly less populated. In the following, we concentrate on the dynamics starting on this particular cationic PES. As seen in Fig. 1, the potential energy surfaces of $X^{2} \Pi_{u}$ and $A^{2} \Sigma_{g}^{+}$approach each other as a function of $\alpha_{a}$, defined as the angle between vectors $\mathrm{C}_{2}-\mathrm{C}_{1}$ and $\mathrm{C}_{1}-\mathrm{H}_{a}$, and $r_{1 a}$, the $\mathrm{C}_{1}-\mathrm{H}_{a}$ bond length. The path to large $\mathrm{CH}$ bond lengths and large $\mathrm{CCH}$ angles is downhill on the $A^{2} \Sigma_{g}^{+}$PES, starting from the ground-state equilibrium acetylene structure. The $X^{2} \Pi_{u}$ ground state of the cation is doubly degenerate in the linear acetylene structure, as seen in Fig. 2(b). In the two-dimensional plot in Fig. 1, only the highest energy $X^{2} \Pi_{u}$ state is shown for the sake of clarity. Two one-dimensional cuts along $\alpha_{a}$ and $r_{1 a}$ are shown as well. In these cuts, the curves of the two $X^{2} \Pi_{u}$ states and the $A^{2} \Sigma_{g}^{+}$state are shown. A conical intersection region between these states is reached for a $\mathrm{CCH}$ angle close to $120 \mathrm{deg}$ and a $\mathrm{CH}$ distance of about $2.2 \AA$. Figure 2(a) shows the PESs of the $X^{2} \Pi_{u}$ and $A^{2} \Sigma_{g}^{+}$ states along the isomerization path projected onto the $\alpha_{a}$ coordinate. Along the cut shown in Fig. 2(a), $r_{1 a}$ is linearly varied from 1.0 to $2.25 \AA$, and the $\mathrm{H}_{b}-\mathrm{C}_{2}-\mathrm{C}_{1}$ angle is kept fixed at $10 \mathrm{deg}$. The seam of conical intersection reached along the isomerization coordinate is located about $1 \mathrm{eV}$ below the acetylene geometry on the $A^{2} \Sigma_{g}^{+}$PES. The conical intersection seam is multidimensional in character,
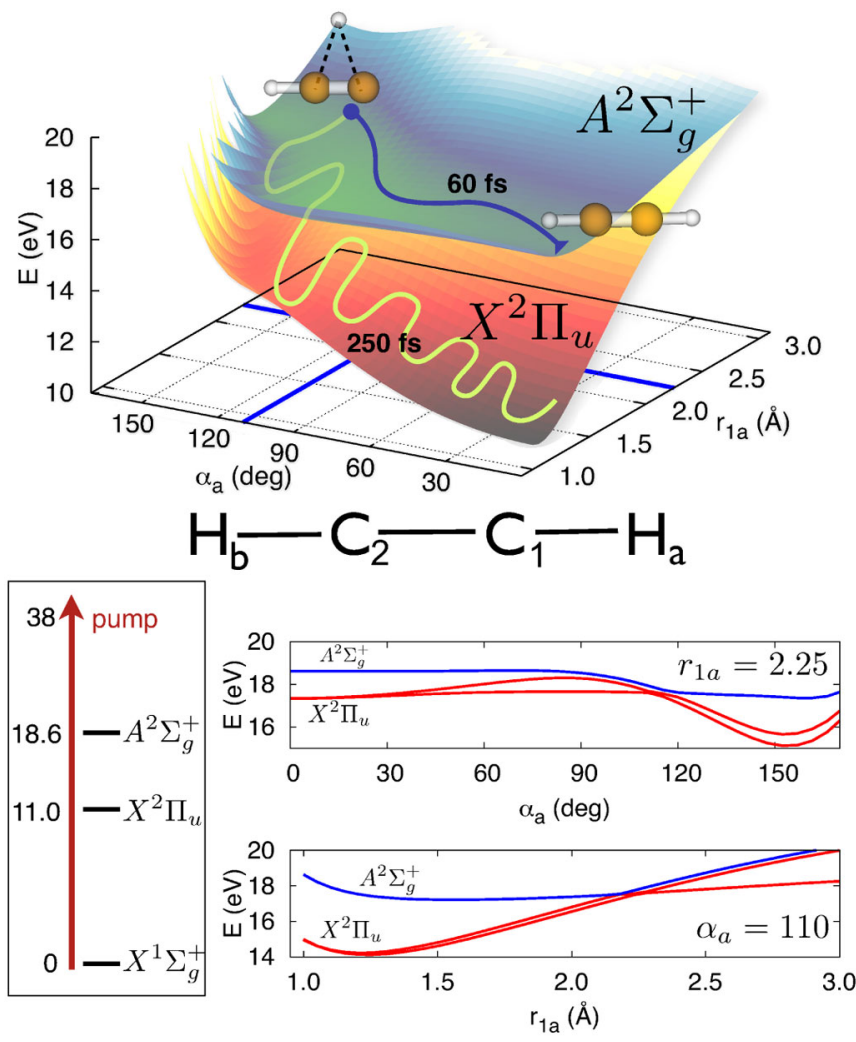

FIG. 1 (color online). Upper part: Potential energy surfaces for the $X^{2} \Pi_{u}$ and $A^{2} \Sigma_{g}^{+}$states of the monocation. Lower part: Two one-dimensional cuts along the $\mathrm{C}_{2}-\mathrm{C}_{1}-\mathrm{H}_{a}$ angle $\left(\alpha_{a}\right)$ and the $\mathrm{C}_{1}-\mathrm{H}_{a}$ distance $\left(r_{1 a}\right)$ for the PESs of the $X^{2} \Pi_{u}$ and $A^{2} \Sigma_{g}^{+}$states. In all curves the rest of coordinates are kept at their ground-state values. 

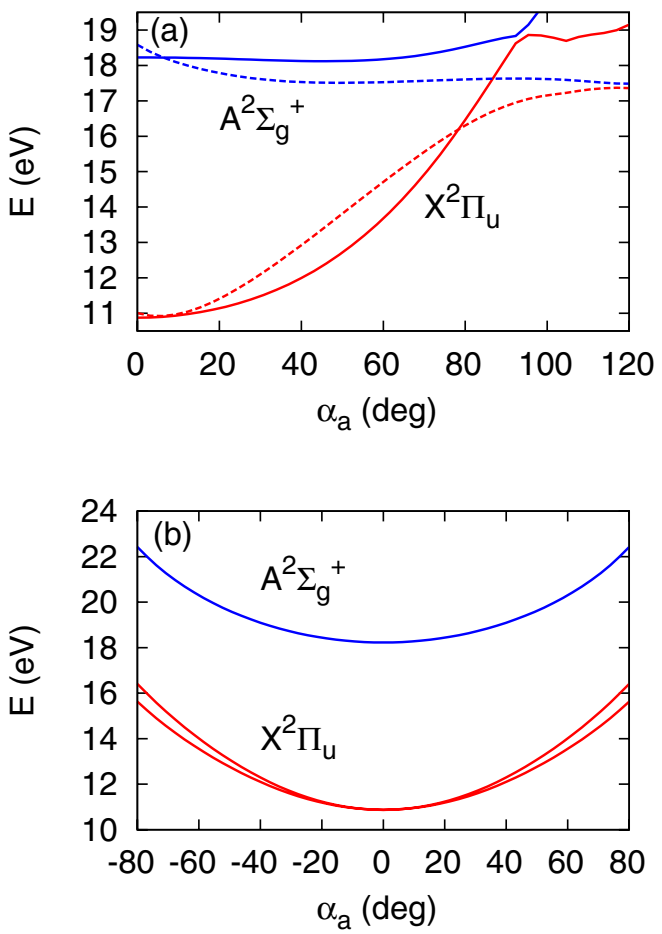

FIG. 2 (color online). (a) Potential energy cuts along the isomerization (dashed lines) and trans-bend (solid lines) paths for the $X^{2} \Pi_{u}$ and $A^{2} \Sigma_{g}^{+}$states as a function of the $\alpha_{a}$ angle. The $\mathrm{C}_{1}-\mathrm{H}_{a}$ distance is linearly varied from 1 to $2.25 \AA$ along the isomerization path. Along the trans-bend path, both $\alpha_{a}$ and $\mathrm{H}_{b}-\mathrm{C}_{2}-\mathrm{C}_{1}\left(\alpha_{b}\right)$ angles are varied by the same amount, and the $\mathrm{H}_{b}-\mathrm{C}_{2}-\mathrm{C}_{1}-\mathrm{H}_{a}$ dihedral angle is set to $180 \mathrm{deg}$. All other coordinates are kept frozen. Only the higher energy $X^{2} \Pi_{u}$ state is shown. (b) Potential energy curves along the cis-bend path. Both $\alpha_{a}$ and $\alpha_{b}$ angles are varied by the same amount, and the $\mathrm{H}_{b}-\mathrm{C}_{2}-\mathrm{C}_{1}-\mathrm{H}_{a}$ dihedral angle is set to $0 \mathrm{deg}$.

and is also reached along the so-called trans-bend coordinate, along which both $\mathrm{CCH}$ angles bend in opposite directions starting from a linear structure [11]. Along this path to the seam of intersection the PES of the $A^{2} \Sigma_{g}^{+}$state is approximately flat, and the intersection is reached at a higher energy than the intersection point along the isomerization path, as seen in Fig. 2(a). Hence, trajectories starting on the $A^{2} \Sigma_{g}^{+}$PES rapidly evolve into vinylidene configurations along a downhill, barrierless path. On their way, they approach the seam of intersection between this state and $X^{2} \Pi_{u}$, where the Born-Oppenheimer approximation breaks down, and the state $X^{2} \Pi_{u}$ gets populated. The intersection region is about $6 \mathrm{eV}$ above the minimum energy configurations on the $X^{2} \Pi_{u}$ PES, which are acetylenelike. Most of the potential energy of the vibrationally hot $X^{2} \Pi_{u}$ state will be initially found in the C-C-H angular coordinates, as can already be inferred by inspecting the 2D surface in Fig. 1. Part of this energy can be redistributed, through an efficient IVR mechanism in the $X^{2} \Pi_{u}$ state, over the various vibrational modes-especially the $\mathrm{C}-\mathrm{C}$ vibrational mode, as will be explained later. This
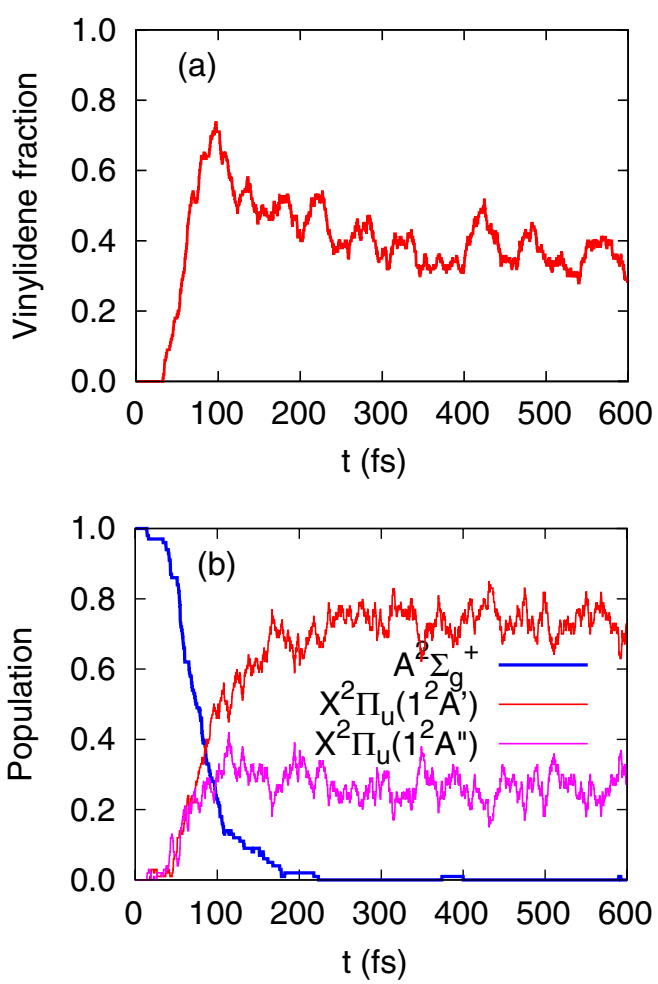

FIG. 3 (color online). (a) Time evolution of the vinylidene fraction when starting from the $A^{2} \Sigma_{g}^{+}$state of the acetylene monocation. (b) Time evolution of the electronic population of the $A^{2} \Sigma_{g}^{+}$and $X^{2} \Pi_{u}$ states for initial preparation of the acetylene cation in the $A^{2} \Sigma_{g}^{+}$state.

energy redistribution brings the system partially back to acetylene configurations in the lower state $X^{2} \Pi_{u}$ and has an onset time of a few hundred femtoseconds. The red curve in Fig. 3(a) shows the vinylidene population as a function of time. Vinylidene configurations are defined here as those in which both hydrogens are found at the same side with respect to the imaginary plane intersecting the midpoint of the $\mathrm{CC}$ bond and perpendicular to it. The vinylidene fraction reaches its maximum of almost 0.8 about $100 \mathrm{fs}$ after ionization. After about $60 \mathrm{fs}$ its value reaches half its maximum, in good agreement with the experimental time of $52 \pm 15 \mathrm{fs}$ reported in Ref. [7]. After the maximum fraction of vinylidene has been reached, it continuously decreases until stabilizing at a value of 0.4-0.5, after about 500 fs. During this time, the system redistributes energy from the isomerization coordinates (mostly the angular coordinates) into other vibrations, and by doing so it partially repopulates acetylenelike conformations on the $X^{2} \Pi_{u}$ state. Recently, a similar effect was observed by Hishikawa et al. [6] in the ultrafast isomerization of deuterated acetylene dication. It is known that the Landau-Zener method overestimates electronic transition rates with respect to other TSH approaches [19]. In the present case, this causes the vinylidene to acetylene relaxation process to be too fast. Measurements 
using a larger pump-probe delay than used in Ref. [7] should be able to detect that relaxation mechanism.

The time evolution of the electronic population of the $A^{2} \Sigma_{g}^{+}$and $X^{2} \Pi_{u}$ states for initial preparation of the acetylene cation in the $A^{2} \Sigma_{g}^{+}$state is shown in the lower part of Fig. 3. During the first tens of femtoseconds, the electronic population remains essentially in the initial $A^{2} \Sigma_{g}^{+}$state. Once the conical intersection is reached, ultrafast population decay of the $A^{2} \Sigma_{g}^{+}$state takes place, accompanied by an ultrafast electronic population of the $X^{2} \Pi_{u}$ states. At around $200 \mathrm{fs}$, the $A^{2} \Sigma_{g}^{+}$state is completely depopulated. One also observes during the first $100 \mathrm{fs}$ a strong correlation between the fast population decay of the $A^{2} \Sigma_{g}^{+}$state and the rapid increase in the vinylidene fraction, pointing to an ultrafast decay through isomerization and conical intersections. After $100 \mathrm{fs}$, while the population in the lower $X^{2} \Pi_{u}$ state is still increasing, the vinylidene fraction is already decreasing, reflecting the start of an effective IVR process. The time evolution of the vinylidene fraction shows that, after electronic relaxation from $A^{2} \Sigma_{g}^{+}$to $X^{2} \Pi_{u}$, vibrational energy redistribution on a time scale of 200300 fs takes place. To better understand this process, the ensemble average of the total vibrational kinetic energy $\left\langle T_{\text {vib }}\right\rangle$ for trajectories starting on the $X^{2} \Pi_{u}$ and $A^{2} \Sigma_{g}^{+}$PESs was computed. $T_{\text {vib }}$ is defined as $T_{\text {tot }}-T_{\text {c.m. }}-T_{\text {rot }}$, where $T_{\text {tot }}$ is the total kinetic energy of the system of particles, $T_{\text {c.m. }}$ is the kinetic energy of the center of mass motion, and $T_{\text {rot }}$ is the rotational energy around the center of mass. For each trajectory at $t=0$, both $T_{\text {c.m. }}$ and $T_{\text {rot }}$ are zero, and remain small at all times within the numerical accuracy of the integration. $\left\langle T_{\text {vib }}\right\rangle$ is shown in Fig. 4. After ionization into the $A^{2} \Sigma_{g}^{+}$state, $\left\langle T_{\text {vib }}\right\rangle$ increases rapidly during the first $200 \mathrm{fs}$ and reaches a plateau value of about $3 \mathrm{eV}$. This increase in average kinetic energy is of course due to the large energy gap of about $7 \mathrm{eV}$ between $X^{2} \Pi_{u}$ and $A^{2} \Sigma_{g}^{+}$ states for acetylene configurations. The final $\left\langle T_{\mathrm{vib}}\right\rangle$ value is about half of the potential energy initially available in the state $X^{2} \Pi_{u}$ from the conical intersection to the minimum, which is consistent with the equipartition theorem. The 200-300 fs needed to reach the plateau value can therefore be seen as a sort of thermalization time needed to redistribute the potential energy among the various degrees of freedom of the system. This rapid increase in $\left\langle T_{\text {vib }}\right\rangle$ is not found when switching off electronic transitions. Moreover, when starting in the $X^{2} \Pi_{u}$ state (see Fig. 4 ), $\left\langle T_{\text {vib }}\right\rangle$ only oscillates slightly because the $X^{2} \Pi_{u}$ PES is not very different from the neutral ground state PES. The average kinetic energy $\left\langle T_{c c}\right\rangle$ of the C-C stretching mode is shown in the inset of Fig. 4. Similarly as for $\left\langle T_{\text {vib }}\right\rangle$, after starting in the $A^{2} \Sigma_{g}^{+}$state, $\left\langle T_{c c}\right\rangle$ increases rapidly in the first $200 \mathrm{fs}$ until it reaches a final plateau of about $0.9 \mathrm{eV}$. Therefore, $\left\langle T_{c c}\right\rangle$ is nearly $1 / 3$ of the total vibrational energy of the system after IVR. Among the six internal vibrational degrees of freedom of the system, the $\mathrm{C}-\mathrm{C}$ mode alone is taking a large amount of the energy, acting as a reservoir

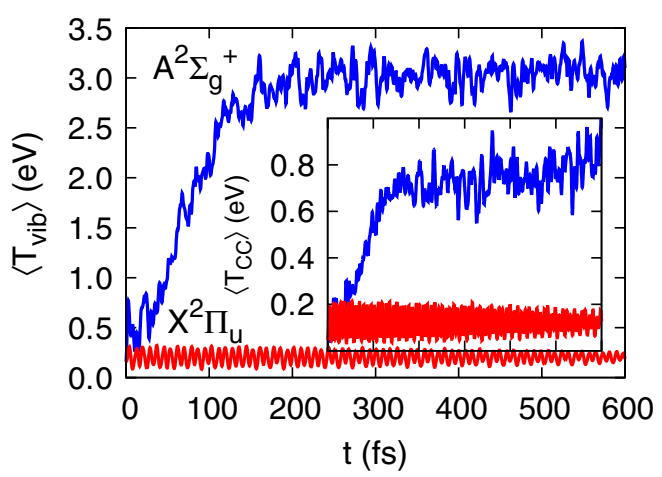

FIG. 4 (color online). Time evolution of the ensemble average of the total vibrational energy for $A^{2} \Sigma_{g}^{+}$and $X^{2} \Pi_{u}$ initial states. The inset presents the evolution of the vibrational energy of the C-C mode.

and cooling down the isomerization coordinates. Simultaneously to the increase in $\left\langle T_{c c}\right\rangle$ during the first $200 \mathrm{fs}$, an increase of $0.2 \AA$ in the average C-C distance is observed.

In conclusion, we have studied the dynamics of acetylene cations after extreme ultraviolet photoionization. We showed that ionization into the $A^{2} \Sigma_{g}^{+}$state is followed by an ultrafast hydrogen migration leading to vinylidene, accompanied by electronic and vibrational energy relaxation. Our model not only describes essential features of the fast decay mechanism of the $A^{2} \Sigma_{g}^{+}$state but also presents a unified view of the two most prominent processes proposed for the explanation of the fast decay of the $A^{2} \Sigma_{g}^{+}$state. We find that ultrafast hydrogen migration goes hand in hand with an ultrafast nonradiative decay of the $A^{2} \Sigma_{g}^{+}$state to the $X^{2} \Pi_{u}$ state via conical intersections. Moreover, we predict that the skeletal carbon-carbon stretching mode can efficiently absorb a large fraction of the excess energy, acting as a reservoir and cooling down the isomerization coordinates. This IVR process is found to be responsible for a partial return to acetylenelike conformations in less than half a picosecond after ionization.

We acknowledge A. Rudenko, S.-K. Son, G. Dixit, and U. Bültemeier for useful discussions.

*oriol.vendrell@cfel.de

[1] D. Polli et al., Nature (London) 467, 440 (2010).

[2] L. R. Khundkar and A. H. Zewail, Annu. Rev. Phys. Chem. 41, 15 (1990).

[3] Y. Yu, M. Nakona, and T. Ikeda, Nature (London) 425, 145 (2003).

[4] O. Vendrell and H.-D. Meyer, Phys. Chem. Chem. Phys. 10, 4692 (2008).

[5] P. Rosmus, P. Botschwina, and J. Maier, Chem. Phys. Lett. 84, 71 (1981).

[6] A. Hishikawa et al., Phys. Rev. Lett. 99, 258302 (2007).

[7] Y. H. Jiang et al., Phys. Rev. Lett. 105, 263002 (2010). 
[8] G. Chambaud, R. Van den Boom, and P. Rosmus, Chem. Phys. Lett. 247, 79 (1995).

[9] C. Cha, R. Weinkauf, and U. Boesl, J. Chem. Phys. 103, 5224 (1995).

[10] M. Perić and B. Engels, Chem. Phys. 238, 47 (1998).

[11] R. Gillen, B. Ostojic, and W. Domcke, Chem. Phys. 272, 1 (2001).

[12] S. Boyé-Péronne, D. Gauyacq, and J. Liévin, J. Chem. Phys. 124, 214305 (2006).

[13] J.E. Reutt et al., J. Chem. Phys. 84, 3022 (1986).
[14] H. Köppel, W. Domcke, and L. Cederbaum, Adv. Chem. Phys. 57, 59 (1984).

[15] F. Krausz and M. Ivanov, Rev. Mod. Phys. 81, 163 (2009).

[16] N. Berrah et al., J. Mod. Opt. 57, 1015 (2010).

[17] A. Rudenko (private communication).

[18] J. C. Tully and R. K. Preston, J. Chem. Phys. 55, 562 (1971).

[19] E. Tapavicza et al., J. Chem. Phys. 129, 124108 (2008).

[20] G. A. Jones, B. K. Carpenter, and M. N. Paddon-Row, J. Am. Chem. Soc. 120, 5499 (1998).

[21] G. Cooper et al., Chem. Phys. 125, 307 (1988). 